\title{
Wilson's disease presenting as rapid eye movement sleep behavior disorder: a possible window to early treatment
}

Doença de Wilson apresentando-se com transtorno comportamental do sono REM: uma janela possível para instituição de tratamento precoce

Gotthard G. Tribl ${ }^{1,2}$, Edson Bor-Seng-Shu', Mateus C. Trindade ${ }^{1}$, Leandro T. Lucato ${ }^{3}$, Manoel J. Teixeira ${ }^{1}$, Egberto R. Barbosa ${ }^{1}$

\begin{abstract}
Objective: To describe characteristics of REM sleep behavior disorder in Wilson's disease. Method: Questionnaire-based interviews (patients and relatives), neurological examinations, two-week prospective dream-diary, video-polysomnography, transcranial sonography, MRI. Results: Four Wilson's disease cases with REM sleep behavior disorder were described; three had REM sleep behavior disorder as initial symptom. All showed mesencephalic tegmental/tectal sonographic hyperechogenicities and two presented ponto-mesencephalic tegmental MRI hyperintensities. Conclusion: This first description of REM sleep behavior disorder in Wilson's disease in literature documents REM sleep behavior disorder as a possible presenting symptom of Wilson's disease and adds further evidence to the parallelism of Parkinson's disease and Wilson's disease in phenotype and brainstem topography, which ought to be further studied. REM sleep behavior disorder has prognostic relevance for neurodegeneration in $\alpha$-synucleinopathies. In Wilson's disease, usefulness of early diagnosis and treatment are already well established. REM sleep behavior disorder in Wilson's disease offers a possible theoretical model for potential early treatment in this extrapyramidal and brainstem paradigm syndrome, previewing the possibility of neuroprotective treatment for REM sleep behavior disorder in "pre-clinical” Parkinson's disease.
\end{abstract}

Keywords: Wilson's disease, parkinsonian syndromes, rapid eye movement sleep behavior disorder, parasomnias, tegmentum.

RESUMO

Objetivo: Descrever características do transtorno comportamental do sono REM (TCSR) na doença de Wilson (DW). Método: Aplicação de entrevistas, vídeo-polissonografia, sonografia transcraniana (STC), ressonância magnética (RM), diário de sonhos. Resultados: Descrevemos quatro casos de DW com TCSR. Três apresentaram o TCSR como primeira manifestação. Todos mostraram hiperecogenicidades mesencefálicas na STC, dois apresentaram hiperintensidades ponto-mesencefálicas na RM. Conclusão: Esta é a primeira descrição do TCSR na DW. Relatamos o TCSR como um sintoma inicial da DW. Acrescentamos prova para o paralelismo entre a doença de Parkinson e DW, com relação aos fenótipos e localização das lesões cerebrais. Nas alfa-sinucleinopatias, o TCSR tem relevância prognóstica quanto à neurodegeneração. Na DW, já conhecemos a importância de diagnóstico e tratamento precoces. O TCSR na DW oferece um modelo para antecipar o tratamento desta síndrome de acometimento dos núcleos basais e tronco, vislumbrando a possibilidade de tratamento neuroprotetor para a fase "pré-clínica" da DP.

Palavras-chave: doença de Wilson, síndromes parkinsonianas, transtorno comportamental do sono REM, parassonias, tegmento.

Wilson's disease (WD) causes tissue copper storage, especially in brain, cornea, and liver. Early stages of metal deposits in basal ganglia and brainstem in WD may be detected by transcranial sonography (TCS $)^{1}$. WD's patients frequently suffer from disorders of sleep ${ }^{2,3,4}$, including altered REM sleep $^{2}$, nevertheless REM sleep behavior disorder (RBD) has not been described in the very broad spectrum of clinical presentation of this disease. We present four cases of early neurological WD with RBD; three of them developed $\mathrm{RBD}$ as their initial manifestation of WD. All four cases show

${ }^{1}$ Divisão de Neurologia e Neurocirurgia, Hospital das Clínicas, Faculdade de Medicina, Universidade de São Paulo, Sao Paulo SP, Brazil; SVA Zurich, Zurich, Switzerland;

${ }^{3}$ Departamento de Radiologia, Hospital das Clínicas, Faculdade de Medicina, Universidade de São Paulo, Sao Paulo SP, Brazil.

Correspondence: Gotthard G. Tribl; Divisão de Neurologia e Neurocirurgia, Hospital das Clínicas, Faculdade de Medicina, Universidade de São Paulo; Av. Dr. Enéas de Carvalho Aguiar, 255 - 5ª andar / Sala 5084 Cerqueira César; 05403-900 São Paulo SP, Brasil; E-mail: gtribl@hotmail.com

Conflict of interest: There is no conflict of interest to declare.

Received 13 May 2014; Received in final form 26 June 2014; Accepted 16 July 2014. 
TCS hyperintensities of midbrain tegmentum, the location considered crucial for the genesis of idiopathic $\mathrm{RBD}^{5,6,7}$ and of RBD in Parkinson's disease (PD) ${ }^{8}$.

The relationship between WD and PD has been an issue of special interest in research on extrapyramidal syndromes, since S. A. Kinnier Wilson stressed already in his seminal first description of WD the considerable parallels between these diseases, both in clinical symptoms and in localization of brain lesions. ${ }^{9}$. Our WD cases, presenting both RBD and tegmental lesions, provide new further evidence for that parallelism, extending it to early disease stages.

Early neuroprotective treatment of RBD in order to avoid or delay neurodegeneration in $\alpha$-synucleinopathies has been one of the major challenges of neuropharmacological research $^{10}$. In WD, highly effective treatment options are available, which allow slowing down or almost stopping the cascade of brain tissue destruction, provided the treatment is applied as soon as any disease activity appears ${ }^{11}$. This opens the opportunity to consider RBD, presenting as an early manifestation of WD, a theoretical model for research in early diagnosis, treatment, and neuroprotection of "pre-clinical" PD.

\section{METHOD}

All cases underwent the following examinations: extensive structured clinical and sleep interviews with the patients and their mothers, complete medication history (with focus on antidepressants and neuroleptics), RBD Questionnaire Hong Kong (RBDQHK), RBD Screening Questionnaire (RBD-SQ), Mayo Sleep Questionnaire (MSQ), Pittsburgh Sleep Quality Index (PSQI), Epworth Sleepiness Scale (ESS), International RLS Study Group Rating Scale (IRLS), Mini Mental State Examination (MMSE), Addenbrooke's Cognitive Examination - Revised (ACE-R), Beck Depression Inventory (BDI), clinical examination, neurological examination with the Unified Wilson's Disease Rating Scale (UWDRS) and the Global Assessment Scale for WD (GAS for WD), two-week dream diary requesting written full length dream reports and asking additional retrospective and prospective questions on dream and nightmare frequencies and on emotional quality of dreams, whole-night video-polysomnography (VPSG; Embla Systems, Remlogic Version 3.2, Thornton, CO, US; six EEG channels, EOG, bilateral EMG of submental, flexor digitorum superficialis and anterior tibial muscles, oronasal thermal sensor, nasal air pressure transducer, thoracic and abdominal inductance plethysmography, pulse oximetry, electrocardiogram, ambiance microphone; PSG evaluation according to American Academy of Sleep Medicine Criteria ${ }^{12}$, determination of REM sleep without atonia (RWA) according to criteria of the SINBAR group $^{13}$ ), TCS (MicroMaxx ${ }^{\mathbb{R}}$ ultrasound system, Sonosite, US), and at least one brain MRI (including multiplanar T1, T2-weighted, SWI and FLAIR images in 1.5T or 3T). Diagnosis of WD was established according to Ferenci ${ }^{14}$, diagnosis of RBD according to ICSD- $3^{15}$. This study was approved by the local ethics committee and written informed consent was obtained from all participants.

\section{CASE REPORTS}

Patient A. A 20-year-old female art student. Since age five, the patient used to speak in sleep and acted out dreams. She suffered from very bad subjective sleep quality, violent movements during sleep and innumerous falls out of bed. To protect against injuries, the mattress had to be put on the floor and another one against the wall. After hepatitis at age nine, pathological values of ceruloplasmin (2.4 mg/dl), urine copper $(233 \mu \mu / 24 \mathrm{~h})$, and serum total copper $(85 \mu \mu / \mathrm{dl})$ were found and diagnosis of WD was established. Because of an allergic reaction to D-penicillamine, WD was treated with trientine $500 \mathrm{mg} / \mathrm{d}$.

At age 15, the situation worsened to a severe recurrent depression with insomnia. Almost every morning she recalled very unpleasant dream content in much detail and had nightmares several times a week. Typically she dreamt of being aggressively insulted and attacked, frequently of being raped. Violent dream enactment behavior (DEB), reported at a frequency of 2-3 times a month, caused frequent bruises and falls out of bed, with waking up while falling. This DEB was fully confirmed in a separate interview with her foster-mother who lives in the same home. A whole-night PSG at age 15 was reported unremarkable, but this PSG was performed without video and RWA was not evaluated. Repeated daytime EEGs showed no abnormalities. Since age 18, the patient has been taking clonazepam $0.5 \mathrm{mg}$ at night, initially on a daily basis, and during the last year at a maximum of three times a week. Symptoms improved, but threatening dreams and DEB persisted.

The neurological examination revealed only a discrete postural tremor on all four limbs and minor neuropsychological deficits (MMSE 30/30; ACE-R 89/100, losses in visualspatial construction and verbal fluency). The laboratory values confirmed $\mathrm{WD}$ (ceruloplasmin $<2 \mathrm{mg} / \mathrm{dL}$, urine copper $300 \mu \mathrm{g} / 24 \mathrm{~h}$, serum total copper $<25 \mu \mathrm{g} / \mathrm{dL}$ ). Liver values were normal apart from slightly elevated transaminases (Child-Pugh A). Sleep quality was very bad (PSQI 14, time in bed 2:00 - 11:00 a.m. with about a third in wake state), symptoms of moderate depression were present (BDI 27, no antidepressant therapy). Filling in a prospective two-week dream diary, she wrote detailed dream contents in seven of 14 mornings and remembered having had dreams without recalling content in further three mornings. Four of these dreams 

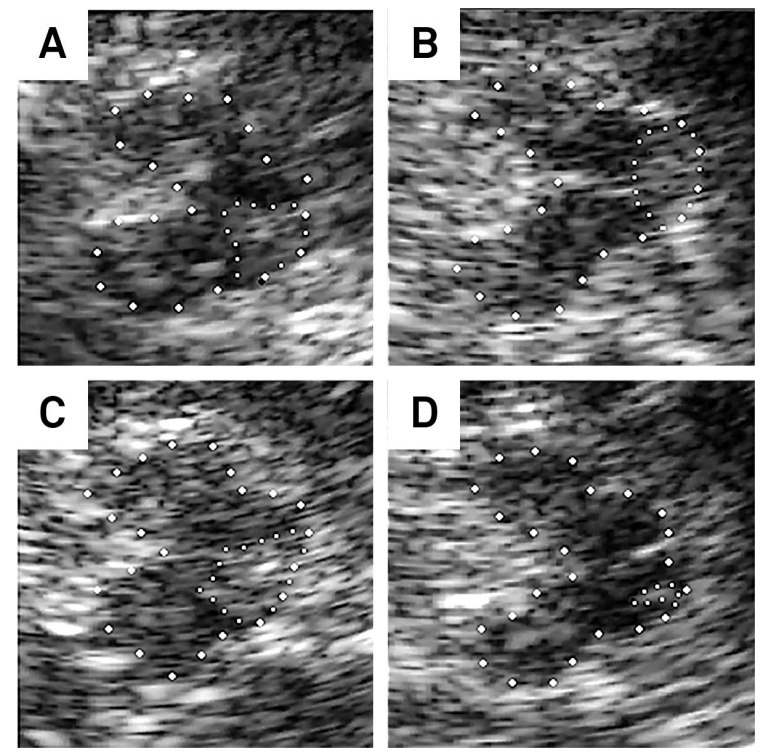

Figure 1. Patients A-D Transcranial sonography of the midbrain (axial section): The hypoechogenic butterfly-shaped midbrain, surrounded by the hyperechogenic basal cisterns, is circled for better visualization (A-D). Hyperechogenic images can be seen in the midbrain tegmentum and/or tectum (also circled).

had explicitly violent content, two of them resulting in injuries (bruises, falling out of bed). 54 of 100 possible points in the RBDQHK further strongly suggested RBD. VPSG (clonazepam withdrawn for six plasma half-lives) showed some loss of sleep periodicity, while the cumulative distribution of sleep stages was preserved. $71,5 \%$ of REM sleep time showing RWA confirmed the diagnosis of $\mathrm{RBD}^{13}$. Video evaluation of REM sleep showed only nine minor movement events of the limbs (myoclonic 5, simple motor 4). Brain MRI was normal (as well as three previous ones since age 12). TCS presented hyperechogenic signs in the midbrain tegmentum and tectum as well as in the substantia nigra (Figure 1; similar TCS findings were present in all four cases).

Patient B. A 31-year-old male pharmaceutical representative. At age 22, the patient developed frequently agitated sleep and repeatedly laughed while sleeping. For the following years and with a frequency of two to three times a month he showed violent DEB, falling out of bed, breaking through his bed base, and hurting his mother in isomorphic self-defense. He easily recalled the threatening dream contents, generally themes of persecution, falling, and being drowned. At age 24, he started to present symptoms of depression and became psychotic. He closed himself up in his room and developed apathy and somnolence. Schizophrenia was diagnosed. Two months later he presented dysphagia, hypersalivation, severe dysarthria, tremor, difficulties in manual object use, leg dystonia and slowing of gait. At age 25, diagnosis of WD was made (ceruloplasmin $<2 \mathrm{mg} / \mathrm{dL}$, total serum copper $13.0 \mu \mathrm{g} / \mathrm{dL}$, presence of Kayser-Fleischer rings). At that time brain MRI showed WD characteristic basal ganglia lesions, and also severe T2/FLAIR diffuse hyperintensity in pontine and mesencephalic tegmentum and mesencephalic tectum (Figure 2). Treatment with D-penicillamine $750 \mathrm{mg} / \mathrm{d}$ was introduced, which had to be changed to trientine $750 \mathrm{mg} / \mathrm{d}$ because of fever reaction. Nevertheless his condition deteriorated further and two years later he became bedridden with generalized dystonia. Bodyweight decreased from $92 \mathrm{~kg}$ to $46 \mathrm{~kg}$ and he had to be fed via a gastrostomy tube. DEB was not observed any longer, while motor handicap increased to almost akinetic parkinsonism and communication became restricted to utterance of simple sounds. A severe insomnia developed. The most recent therapy was trientine $750 \mathrm{mg} / \mathrm{d}$, biperiden $6 \mathrm{mg} / \mathrm{d}$, baclofen $30 \mathrm{mg} / \mathrm{d}$, trazodone $50 \mathrm{mg} / \mathrm{d}$, zolpidem $10 \mathrm{mg}$ at night, cervical botulinum toxin injections, and omeprazole $20 \mathrm{mg} / \mathrm{d}$.

At the neurological examination the patient was functional quadriplegic in almost fixed dystonic positions with severe resting as well as postural tremor of head and extremities. He was bedridden, underweight (BMI 17.5), incapable to communicate and fully dependent in the activities of daily living (ADL). Liver enzymes were normal. Sleep quality was disastrous; he had a sleep latency of hours and would not reach sleep without hypnotics (PSQI 15). VPSG
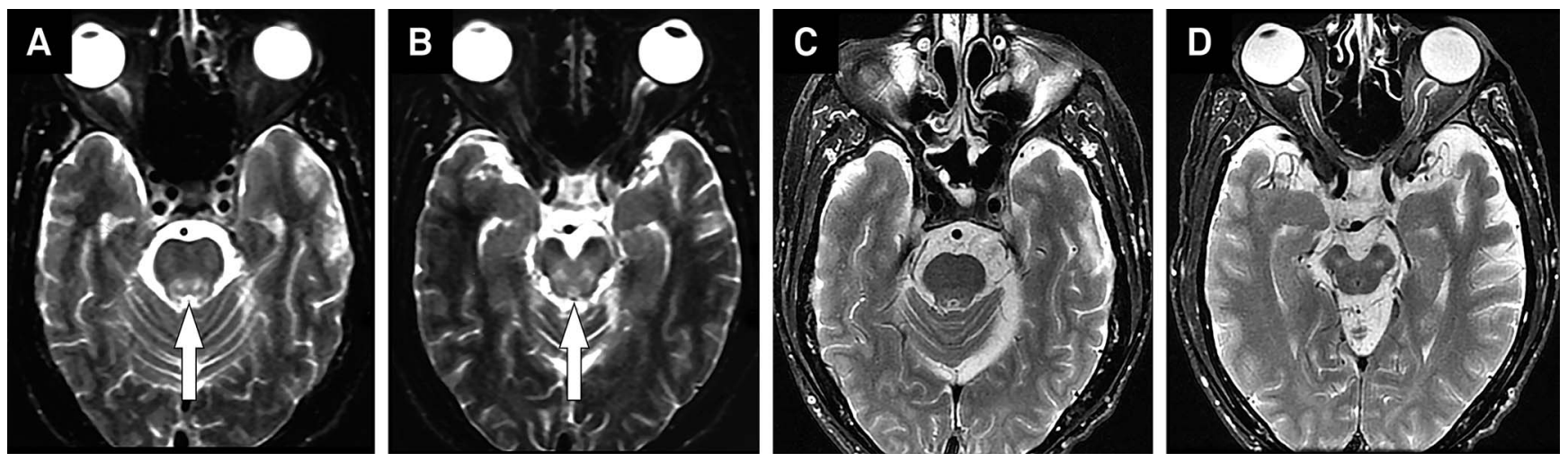

Figure 2. Patient B MRI (axial T2-weighted images): First MRI discloses severe diffuse hyperintensity in pontine tegmentum and mesencephalic tegmentum/tectum (arrows) (A, B). After seven years, lesions were less marked in a follow-up MRI (C, D). 
showed severely reduced sleep efficiency (56.8\%) and deep sleep (stage N3 5.4\%), and $46.4 \%$ of RWA, without motor equivalent on the video. The permanent dystonic tremor ceased while asleep. A follow-up brain MRI, seven years after the first one, showed generalized atrophy, a more evident atrophy of involved structures (basal ganglia and brainstem), evident abnormal metal deposits in red nuclei and substantia nigra, but T2/FLAIR hyperintensities in pons and mesencephalon were less marked (Figure 2).

Patient C. A 40-year-old housewife, former psychology student. Since childhood she presented somniloquy, sometimes aggressively in mode and content. She described her sleep as generally unquiet, having very vivid and easily recalled dreams as well as repeatedly nightmares with themes of fights and aggression, which she acted out, having already fallen out of bed. One year after her sister had died of a fulminant hepatitis, the patient developed an icterus at age 15. WD was diagnosed with low serum total copper $(<10 \mu \mathrm{g} / \mathrm{dL})$ and ceruloplasmin $(<2 \mathrm{mg} / \mathrm{dL})$, and presence of Kayser-Fleischer corneal rings. Treatment with D-penicillamine $750 \mathrm{mg} / \mathrm{d}$ was initiated. Two years later, she developed a task specific dystonia (writer's cramp). Due to subjective intolerance (gastritis and headache) treatment was changed to $150 \mathrm{mg}$ zinc daily (as sulfate) at age 35. Brain MRI at that age was unremarkable. Three years later, a painful, focal, cervical dystonia emerged, which consequently had to be treated with botulinum toxin. She had never taken any antidepressant or antipsychotic medication.

Neurological examination found slight hypomimia, discrete head tremor, neck rigidity, moderate cervical dystonia, low grade bradykinesia of fine finger movements, postural tremor of four limbs, low grade dystonia of trunk and extremities, some gait ataxia, and minor cognitive deficits (MMSE 27/30, ACE-R 85/100 with losses in concentration, memory, verbal fluency, and visuo-spatial construction). Liver enzymes were normal except for a slightly increased alanine-aminotransferase. Sleep quality was reduced
(PSQI 7) and she complained of excessive daytime sleepiness (ESS 15), both due to a behaviorally induced sleep deficiency syndrome and a moderate to severe RLS (started at age 31, worsened during pregnancy, IRLS 21). She reported a dream recall frequency of at least three times a week and one to two nightmares a week. In a two-week dream diary she wrote contents of two nightmares. VPSG found $34.1 \%$ of RWA and 25 short motor events during REM sleep (myoclonic 7 , simple motor 14 , complex motor 4; the most prominent ones performing big circulating leg movements).

Patient D. A 27-year-old female environmental technology student. At age 24, she developed severe dysarthria, dysphagia, hypersalivation and choking, as well as incapacity to walk. Two months later a severe psychiatric syndrome emerged with agitation, aggressiveness, crying and insomnia. She lost $20 \mathrm{~kg}$ of body weight and had to be fed with a nasogastric tube. Low total serum copper $(<8 \mu \mathrm{g} / \mathrm{dL})$ and ceruloplasmin $(3 \mathrm{mg} / \mathrm{dL})$ as well as presence of Kayser-Fleischer rings established the diagnosis of $\mathrm{WD}$. The patient was treated with D-penicillamine $750 \mathrm{mg} / \mathrm{d}$. For the next two years, psychopathology improved to a moderate depression, insomnia did not cease. The also persistent dysarthria made her speech highly unintelligible during daytime. But her mother, sleeping in the same room, reported that she still spoke loud and clearly while sleeping, with a frequency of about once a week. The most recent therapy regime was D-penicillamine $750 \mathrm{mg} / \mathrm{d}$, vitamin B6 $50 \mathrm{mg} / \mathrm{d}$, L-dopa plus benserazide $200 / 50 \mathrm{mg} / \mathrm{d}$, amitriptyline $75 \mathrm{mg} / \mathrm{d}$, propranolol $240 \mathrm{mg} / \mathrm{d}$, and omeprazole $20 \mathrm{mg} / \mathrm{d}$.

Neurological examination found her wheelchairdependent due to generalized dystonia, rigidity and bradykinesia, and severely restricted in all ADL. She presented hypomimia and severe oromandibular dystonia and spoke slowly and with heavy dysarthria. Besides bradyphrenia clear cognitive deficits were present (MMSE 29/30, ACE-R $85 / 100$ with losses in verbal fluency and visuo-spatial orientation). Symptoms of depression and anxiety were evident (BDI 23/63, on treatment with amitriptyline). She complained
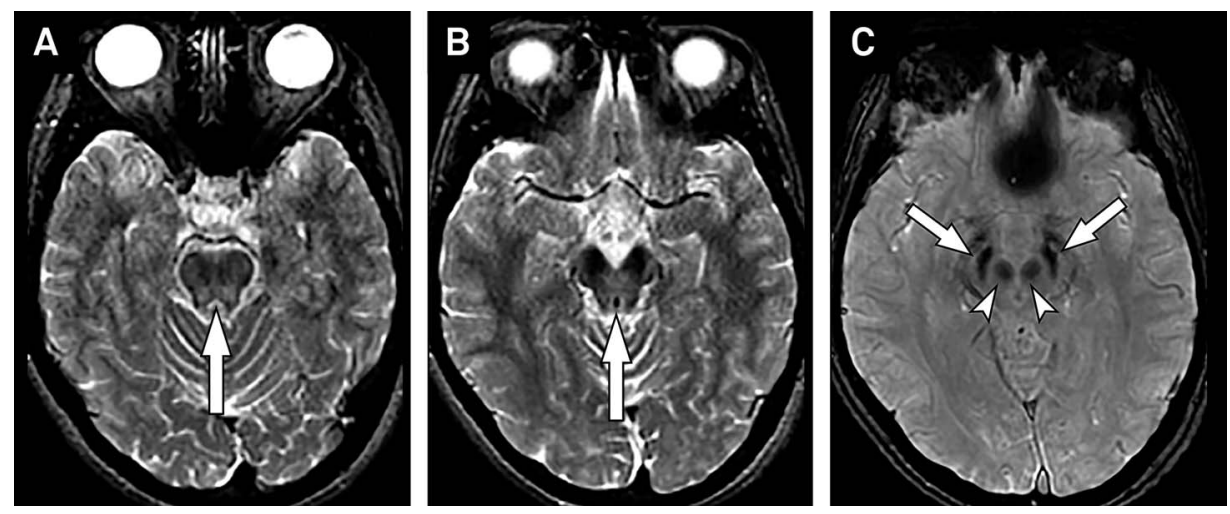

Figure 3. Patient D MRI: MRI (axial T2-weighted images) shows hyperintensity in pontine/mesencephalic tegmentum, and also a less severe involvement of the mesencephalic tectum (arrows) (A, B). Axial SWI demonstrates clearly abnormal hypointensity in substantia nigra (arrows) and red nuclei (arrowheads) (C). 
of insomnia and bad sleep quality (PSQI 10). She reported having very vivid dreams a few times a week and frequently nightmares. In a two-week dream diary she reported four dreams, two of them nightmares, in one of them screaming repeatedly out of horror and then waking up heavily screaming (immediately oriented, dream content of her dead pet cat in decomposition clearly remembered, shouting confirmed by her mother). Liver ultrasound showed compensated, low-grade cirrhosis without ascites. Gastroscopy revealed esophageal varices. Platelet count was $84 \mathrm{mil} / \mathrm{mm}^{3}$, but liver enzymes were normal (Child-Pugh A). VPSG (amitriptyline, L-dopa/benserazide and propranolol withdrawn for six half-lives) showed $19 \%$ of RWA; video documented eight minor movement events of the extremities as motor equivalents (myoclonic 5, simple motor 3 ).

Besides moderate diffuse brain atrophy and WD characteristic basal ganglia lesions (presenting a mixture of T2/FLAIR hyperintensity due to edema/gliosis and T2/SWI (susceptibility-weighted image) hypointensity due to metal deposits), brain MRI showed symmetric SWI hypointensity in red nuclei and substantia nigra (also signaling abnormal metal deposits), and some T2/FLAIR hyperintensity in pontine and mesencephalic tegmentum and mesencephalic tectum (Figure 3).

\section{DISCUSSION}

To our knowledge, we describe the first cases of RBD in $\mathrm{WD}$ in literature. RBD even as initial symptom of WD in three of our cases adds a new facet to the notorious diverse spectrum of possible initial presentations of this disease. In $\mathrm{WD}$, bad sleep quality ${ }^{3}$, excessive daytime sleepiness, poor nocturnal sleep and significantly higher RBD questionnaire scores have been documented ${ }^{2} ; 47.3 \%$ of WD patients had an RBD score in the pathologic range ${ }^{2}$.

All four cases showed midbrain tegmentum and/or tectum hyperechogenicity in TCS; while in only two of them T2/FLAIR MRI disclosed changes in pontine and midbrain tegmentum, possibly due to different physical principles of both techniques. Previous reports have already described a lack of correlation between clinical presentation and MRI findings in $\mathrm{WD}^{16}$.

The MRI changes in our cases are not specific, but probably reflect edema, necrosis, demyelination and gliosis. It is well known that T2/FLAIR-hyperintense MRI lesions in WD may improve after treatment, probably indicating resolution of edema and/or demyelination, as seen in patient B (Figure 2) $)^{17}$.

The exact cause of $\mathrm{RBD}$ is unknown, but pontine and midbrain tegmentum has been discussed as the probable location of origin ${ }^{7}$. In $\boldsymbol{\alpha}$-synucleinopathies, RBD is a highly frequent early stage manifestation of neurodegeneration $^{18}$. WD is a disease of metabolism; but it shares with PD not only the clinical phenomenology but also a similar topographical distribution of basal nuclei and brainstem lesions and the accumulation of metals in these locations ${ }^{1}$. The tegmental lesions in MRI and TCS do not prove a necessary causal connection between $\mathrm{WD}$ and $\mathrm{RBD}$, as it is already established in $\mathrm{PD}$ and $\mathrm{RBD}^{10}$. But the presented cases provide evidence for both RBD as a phenomenon in early neurodegeneration, also in WD, and for tegmental lesions as a possible causal factor for RBD. Further data are necessary to determine the relation between WD and RBD and the possible causal role of tegmental lesions for RBD, both in WD as well as in other parkinsonian syndromes.

However, our cases of WD with RBD and tegmental lesions offer a possible extrapyramidal model with early brainstem manifestation that responds to early neuroprotective treatment. As such it could be of further interest in the recent efforts for development of symptomatic and neuroprotective therapy in RBD as early manifestation of $\mathrm{PD}$ and related syndromes ${ }^{10}$.

Awareness of the possibility of RBD as presenting clinical symptom of WD allows for early diagnosis of special cases. This is of high prognostic importance, as causative, highly effective copper eliminating pharmacotherapy is available and any delay in the beginning of this neuroprotective treatment is the most dangerous constellation for the long-term outcome in $\mathrm{WD}^{11}$.

\section{References}

1. Svetel M, Mijajlovic M, Tomic A, et al. Transcranial sonography in Wilson's disease. Parkinsonism Relat Disord 2012;18:234-238.

2. Nevsimalova S, Buskova J, Bruha R, et al. Sleep disorders in Wilson's disease. Eur J Neurol 2011;18:184-190.

3. Netto AB, Sinha S, Taly AB, et al. Sleep in Wilson's disease: questionnaire based study. Ann Indian Acad Neurol 2011;14:31-34.

4. Portala K, Westermark K, Ekselius L, et al. Sleep in patients with treated Wilson's disease. A questionnaire study. Nord J Psychiatry 2002;56:291-297.

5. Boeve BF, Silber MH, Saper CB, et al. Pathophysiology of REM sleep behaviour disorder and relevance to neurodegenerative disease. Brain 2007;130:2770-2788.
Scherfler C, Frauscher B, Schocke M, et al. White and gray matter abnormalities in idiopathic rapid eye movement sleep behavior disorder: a diffusion-tensor imaging and voxel-based morphometry study. Ann Neurol 2011;69:400-407.

7.

Boeve BF. REM sleep behavior disorder: updated review of the core features, the REM sleep behavior disorder-neurodegenerative disease association, evolving concepts, controversies, and future directions. Ann N Y Acad Sci 2010;1184:15-54.

8. Garcia-Lorenzo D, Longo-Dos Santos C, Ewenczyk C, et al. The coeruleus/subcoeruleus complex in rapid eye movement sleep behaviour disorders in Parkinson's disease. Brain 2013;136:2120-2129. 
Wilson SAK. Progressive lenticular degeneration: a familial nervous disease associated with cirrhosis of the liver. Brain 1912;34:295-507 (p. 479).

10. Schenck CH, Montplaisir JY, Frauscher B, et al. Rapid eye movement sleep behavior disorder: devising controlled active treatment studies for symptomatic and neuroprotective therapy - a consensus statement from the International Rapid Eye Movement Sleep Behavior Disorder Study Group. Sleep Med 2013;14:795-806.

11. European Association for the Study of the Liver. EASL Clinical Practice Guidelines: Wilson's disease. J Hepatol 2012;56:671-685.

12. Berry RB, Brooks R, Gamaldo CE, et al. The AASM Manual for the Scoring of Sleep and Associated Events: Rules, Terminology and Technical Specifications, Version 2.0.3. Darien, Illinois: American Academy of Sleep Medicine, 2014.

13. Frauscher B, Iranzo A, Gaig C, et al. Normative EMG values during REM sleep for the diagnosis of REM sleep behavior disorder. Sleep 2012;35:835-847.
14. Ferenci P, Caca K, Loudianos G, et al. Diagnosis and phenotypic classification of Wilson disease. Liver Int 2003;23:139-142.

15. American Academy of Sleep Medicine. International classification of sleep disorders, $3^{\text {rd }}$ ed. Darien, IL: American Academy of Sleep Medicine; 2014.

16. van Wassenaer-van Hall HN, van den Heuvel AG, Jansen GH, et al. Cranial MR in Wilson disease: abnormal white matter in extrapyramidal and pyramidal tracts. AJNR Am J Neuroradiol 1995;16:2021-2027.

17. da Costa Mdo D, Spitz M, Bacheschi LA, et al. Wilson's disease: two treatment modalities. Correlations to pretreatment and posttreatment brain MRI. Neuroradiology 2009;51:627-633.

18. Iranzo A, Tolosa E, Gelpi E, et al. Neurodegenerative disease status and post-mortem pathology in idiopathic rapid-eye-movement sleep behaviour disorder: an observational cohort study. Lancet Neurol 2013;12:443-453. 